\title{
Evidências de Validade da Escala Indicadores das Características do Ambiente Organizacional (ICAO)
}

\author{
Paula Andréa Prata Ferreira ${ }^{1, *}$, Clarissa Pinto Pizarro de Freitas ${ }^{2}$, \\ Rita Pimenta de Devotto ${ }^{3}$, Bruno Figueiredo Damásio ${ }^{4}$
}

\begin{abstract}
http:/ / orcid.org/0000-0002-1526-491X / Universidade Estácio de Sá, Brasil
${ }^{2}$ http://orcid.org/0000-0002-2274-8728 / Pontifícia Universidade Católica do Rio de Janeiro (PUCRJ), Brasil

${ }^{3}$ http://orcid.org/0000-0002-2287-2526 / Pontifícia Universidade Católica de Campinas (PUC-Campinas), Brasil

${ }^{4}$ http://orcid.org/0000-0002-1150-092X / Universidade Federal do Rio de Janeiro (UFRJ), Brasil
\end{abstract}

\section{Resumo}

As evidências de validade das escalas de Indicadores das Características do Ambiente Organizacional (ICAO) nas versões longa (35 itens) e breve (25 itens) foram avaliadas dentro do contexto brasileiro. A pesquisa utilizou duas amostras, sendo que a primeira foi composta por 1.325 profissionais $(63,8 \%$, sexo feminino), com idade média de 39,9 anos $(D P=11,9)$. A segunda amostra foi formada por 559 profissionais $(55,1 \%$ sexo feminino) com idade média de 43,5 anos $(D P=12)$. A análise fatorial confirmatória demonstrou que a solução de sete fatores de primeira ordem se constitui como uma solução adequada para as duas versões da ICAO. A auto-eficácia ocupacional, engajamento no trabalho e sentido do trabalho apresentaram relações positivas com os recursos do trabalho, porém, negativas com a demanda. Os resultados sugerem o uso da ICAO-25 em detrimento da ICAO-35, devido aos índices de ajustes superiores da ICAO-25, assim como pela brevidade da respectiva escala de indicadores.

Palavras-chave: condições de trabalho, análise fatorial, stress ocupacional.

Evidence of Validity of the Indicator for Characteristics of the Organizational Environment Scale (ICOES)
Evidencias de validez de la Escala Indicadores de las Características del Entorno Organizacional (ICEO)
Abstract

The evidence of validity of the Indicators for Characteristics of the Organizational Environment Scale (ICOES) in the long (35 items) and short ( 25 items) versions were evaluated for the Brazilian context. The survey consisted of two samples, the first being 1,325 professionals $(63.8 \%$, female), with an average age of 39.9 years $(S D=11.9)$. The second sample was composed of 559 professionals (55.1\% female) with an average age of 43.5 years $(S D=12)$. Confirmatory factor analysis showed that the first-order seven-factor solution is adequate for both versions of the ICOES. Occupational self-efficacy, work engagement, and meaning of the work showed positive relationships with job resources, and negative with demand. The results suggest the use of ICOES-25 to the detriment of ICOES-35, due to the superior adjustment indices of ICOES-25, as well as the brevity of the scale.

Keywords: working conditions, factor analysis, occupational stress

\section{Resumen}

La evidencia de validez de las escalas del Indicadores de las Características del Entorno Organizacional (ICEO) en las versiones larga (35 ítems) y corta (25 ítems) fueron investigadas dentro del contexto brasileño. La encuesta utilizó dos muestras, la primera fue composta 1.325 profesionales $(63,8 \%$, mujeres $)$, con una edad promedio de 39,9 años $(D P=11,9)$. La segunda se formó de 559 profesionales $(55,1 \%$ mujeres) con una edad media de 43,5 años $(D P=12)$. El análisis factorial confirmatorio demostró que la solución de siete factores de primer orden es una solución adecuada para ambas versiones de la ICEO. La auto eficacia ocupacional, entusiasmo por el trabajo y sentido del trabajo mostraron relaciones positivas con los recursos de trabajo, sin embargo, negativas con la demanda. Los resultados sugieren el uso de ICAO-25 en detrimento de ICAO-35, debido a los mejores índices de ajuste de ICAO-25, así como a la brevedad de la escala de indicadores.

Palabras-claves: condiciones de trabajo, análisis factorial, estrés ocupacional.
Como citar esse artigo:

Prata-Ferreira, P. A., Freitas, C. P. P., Devotto, R. P., \& Damásio, B. F. (2020). Evidências de Validade da Escala Indicadores das Características do Ambiente Organizacional (ICAO). Revista Psicologia: Organizaçōes e Trabalbo, 20(3), 1141-1149. https://doi.org/10.17652/ 
Observa-se que um ambiente organizacional composto por características que tornem viáveis a realização do trabalho é algo necessário para que os profissionais mantenham seu desempenho e bem-estar no contexto organizacional (Bakker \& Demerouti, 2017; Costa, Borges, \& Barros, 2015; Gouvêa, 2016). A fim de compreender os fatores relacionados ao contexto do trabalho, a teoria de demandas e recursos do trabalho (Job Demand-Resources Theory, JD-R) foi proposta como uma estratégia para pesquisar o modo como demandas e recursos do trabalho e recursos pessoais impactam, direta ou indiretamente, no bem-estar dos trabalhadores, no seu comprometimento com a organização e no seu desempenho laboral (Bakker \& Demerouti, 2018; Demerouti, Bakker, Nachreiner, \& Schaufeli, 2001). Desse modo, as condições de trabalho podem ser categorizadas como demandas do trabalho e recursos do trabalho (Bakker \& Demerouti, 2014; Bakker, Demerouti, \& Sanz-Vergel, 2014; Schaufeli \& Taris, 2014).

Os recursos do trabalho referem-se às características físicas, sociais ou organizacionais do trabalho que podem (a) promover meios para a obtenção de metas, (b) reduzir demandas do trabalho, bem como diminuir os custos fisiológicos e psicológicos associados às demandas do trabalho e, por fim, (c) estimular o crescimento e desenvolvimento pessoal (Demerouti et al., 2001). A autonomia (grau de controle sobre o próprio trabalho), apoio dos colegas, clareza de papel, como também o feedback são exemplos de recursos do trabalho (Bakker, Demerouti, de Boer, \& Schaufeli, 2003; Demerouti \& Bakker, 2011). Os recursos do trabalho podem ser classificados de quatro formas: recursos referentes à organização, ao grupo de trabalho, à forma de organização da tarefa, como também à tarefa em si (Bakker \& Demerouti, 2007, 2018; Nahrgang, Morgeson, \& Hofmann, 2011; Schaufeli, 2017).

Aspectos físicos, sociais ou organizacionais do trabalho que exigem esforços físicos ou mentais referem-se às demandas do trabalho e, por conseguinte, acarretam custos físicos e psicológicos, como também geram tensões. Desse modo, as demandas do trabalho se encontram associadas a custos psicológicos como a exaustão emocional. Podem estar associadas, também, as condições ambientais físicas penosas como, por exemplo, de que maneira a carga de trabalho é distribuída e exigida, a ambiguidade de papéis, pressão de tempo (Crawford, LePine, \& Rich, 2010; Hakanen \& Roodt, 2010), os conflitos interpessoais (Schaufeli \& Taris, 2014). Em uma revisão crítica da teoria JD-R realizada por Schaufelli e Taris (2014), o assédio moral é caracterizado como uma demanda do trabalho.

Ressalta-se que as demandas do trabalho podem variar conforme a função laboral exercida (Schaufeli \& Taris, 2014). Assim, a sobrecarga física tem maior destaque para um trabalhador da construção civil, ao passo que as demandas cognitivas têm maior proeminência para profissionais que exercem atividades de cunho científico, como profissionais da tecnologia da informação, como também as demandas emocionais para profissionais que trabalham em contato frequente com clientes. Ademais, as demandas do trabalho contribuem para explicar os altos níveis de exaustão física e emocional, queixas psicossomáticas e lesões por esforço repetitivo (Bakker et al., 2003; Hakanen, Bakker, \& Schaufeli, 2006).

O desgaste físico, psicológico e/ou emocional são características intrínsecas das demandas do trabalho (Bakker \& Demerouti, 2018). Apesar disso, as demandas podem ser diferenciadas como demandas desafiadoras e demandas de obstáculos. As demandas desafiadoras do trabalho são aquelas que colaboram para o crescimento e aprimoramento do trabalhador (Bakker \& Demerouti, 2014; Crawford et al., 2010). As demandas de obstáculo são identificadas como demandas que prejudicam o desenvolvimento pessoal, a aprendizagem e o alcance de objetivos (Bakker \& Demerouti, 2014; Crawford et al., 2010).

Os recursos pessoais foram integrados à teoria JD-R em um momento posterior (Schaufeli \& Taris, 2014) e tem como função colaborar em prol da adequação de diversas situações e contingências do ambiente. São intrínsecos e se referem a forma com que o trabalhador percebe sua capacidade em transformar positivamente seu ambiente laboral (Xanthopoulou, Bakker, Demerouti, \& Schaufeli, 2007). A autoeficácia, o otimismo e a personalidade proativa são exemplos de recursos pessoais (Schaufeli \& Taris, 2014).

Além de possibilitar que as características de diferentes contextos organizacionais sejam categorizadas como demandas do trabalho e recursos do trabalho, a teoria JD-R compreende que a interação das condições de trabalho pode promover o desenvolvimento do processo motivacional ou do processo de comprometimento da saúde (Bakker \& Demerouti, 2017; Schaufeli \& Taris, 2014). A presença de altos índices de recursos do trabalho e pessoais podem desempenhar um papel motivacional extrínseco ao diminuir o impacto negativo das demandas de trabalho e um papel intrínseco ao satisfazer necessidades humanas, como o relacionamento interpessoal. O processo de motivação está associado a maiores níveis de comprometimento organizacional, ao engajamento no trabalho, baixos índices de despersonalização e excelente desempenho laboral (Bakker \& Demerouti, 2018; Schaufeli, 2017; Schaufeli \& Taris, 2014).

Por outro lado, profissionais expostos a extensos períodos com altos índices de demandas, para os quais eles não têm oportunidade de se recuperar adequadamente, podem vivenciar o processo de comprometimento da saúde. Esse decurso pode levar ao esgotamento físico, mental e/ou emocional e está associado ao desenvolvimento do burnout, tensão ocupacional e adoecimento do profissional (Bakker \& Demerouti, 2014; Schaufeli \& Taris, 2014).

O impacto das condições de trabalho sobre os níveis de bem-estar, saúde mental e física, como também o desempenho dos profissionais tem motivado o desenvolvimento de diversos estudos para compreender a interação dos recursos e das demandas do trabalho, assim como o desenvolvimento dos processos motivacionais e de comprometimento da saúde (Bakker \& Demerouti, 2018; Schaufeli, 2017; Schaufeli \& Taris, 2014). Frente a esse cenário, observa-se a relevância de serem utilizados instrumentos confiáveis e fidedignos para avaliar os recursos e demandas do trabalho.

O Health and Safety Executive's Management Standards Indicator Tool - HSEMSI (Cousins et al., 2004) comissionado pelo Health and Safety Executive (HSE) do Reino Unido, é um instrumento que avalia sete fatores psicossociais no ambiente de trabalho (controle, apoio social do supervisor, apoio social dos colegas, papel, mudança, assédio moral e demanda). O HSEMSI tem sido utilizado na Europa para medição e redução do estresse no trabalho, bem como um indicador para orientar o desenvolvimento de intervenções com maior ênfase na prevenção (melhoria da saúde, bem-estar e motivação) (Toderi \& Balducci, 2015). As sete dimensões do instrumento indicam os fatores psicossociais, que quando deficitários ou ausentes, podem causar estresse no trabalho. Por outro lado, quando os estes fatores psicossociais são otimizados, eles podem determinar um ambiente de trabalho saudável, com motivação e alto desempenho (Toderi \& Balducci, 2015).

A versão original do HSEMSI, em inglês, encontra-se adaptada para 18 línguas. O estudo de Edwards e Webster (2012), envolvendo 67.347 profissionais britânicos, demonstrou que a solução de sete fatores de primeira ordem constituía-se como uma solução adequada para a ICAO-35 $\left(\chi^{2}(537)=119.301,96 ; p<0,01\right.$; CFI $=0,91, \mathrm{GFI}=0,91$; NFI $=0,91$ e RMSEA $=0,05)$ e ICAO-25 $\left(x^{2}(254)=54.800,22 ; p<0,01 ; \mathrm{CFI}=0,94, \mathrm{GFI}=0,94\right.$; NFI $=$ $0,94$ e RMSEA $=0,05)$. Além disso, foi observado que a estrutura foi invariante para a ICAO-35 e ICAO-25 entre profissionais britânicos do setor público e privado, como também para empresas de pequeno, médio e grande porte (Edwards \& Webster, 2012). 
O estudo de validação da versão de 35 itens do HSEMI para a Itália (Toderi et al., 2013) utilizou duas amostras de trabalhadores de organizações do setor privado, sendo 1.298 funcionários italianos e uma amostra de 7.589 funcionários do Reino Unido. Este estudo produziu mais evidências sobre a robustez da solução de 7 fatores do HSEMI em dados do Reino Unido $\left(\chi^{2}(539)=15.321,25\right.$; $p<0,01$; CFI $=0,96$ e RMSEA $=0,06)$ e os índices de confiabilidade das dimensões foram adequados, variando de 0,77 a 0,87 . Foi observado que a solução de sete fatores era adequada ao contexto italiano $\left(\chi^{2}(539)=2.772,48 ; p<0,01\right.$; CFI $=0,96$ e RMSEA $=$ $0,06)$, apresentando valores de consistência interna satisfatórios nas dimensões, com valores de 0,72 a 0,85 . Os achados demonstraram também que a escala apresentava invariância configural, métrica e escalar entra os profissionais italianos e ingleses. Esses achados corroboram que este instrumento é recomendado para a triagem de riscos psicossociais nos locais de trabalho italianos.

Toderi e Balducci (2015) investigaram a relação entre os níveis de controle, apoio social do supervisor, apoio social dos colegas, papel, mudança e demanda com o desempenho, desenvolvimento pessoal, altruísmo e cidadania organizacional. Foi observado que os índices de demanda estavam negativamente relacionados ao desenvolvimento pessoal, e os recursos do trabalho positivamente associados ao desenvolvimento pessoal, altruísmo e cidadania organizacional. As relações encontradas ampliam a rede nomológica do questionário e fornecem evidências empíricas à noção de "business case" para prevenção do estresse no trabalho. Esses resultados sugerem que as organizações com um ambiente de trabalho psicossocial com baixa disponibilidade de recursos e altos níveis de demanda podem esperar não apenas resultados negativos relacionados ao estresse no trabalho, mas também prejuízos no desempenho e desenvolvimento dos profissionais.

Com o objetivo de disponibilizar um instrumento confiável e fidedigno para avaliar os recursos e demandas do trabalho no Brasil, o presente estudo buscou adaptar e avaliar as evidências de validade do Indicador das Características do Ambiente Organizacional ICAO (Health and Safety Executive's Management Standards Indicator Tool - HSEMSI) (Cousins et al., 2004), na versão de 35 itens e na versão de 25 itens da ICAO, de forma a oferecer um instrumento preciso e confiável para uso nacional. As versões de 35 e 25 itens avaliam os recursos do trabalho identificados como controle, apoio social do supervisor, apoio social dos colegas, papel, mudança, assim como os níveis de assédio moral e demanda (Cousins et al., 2004).

A dimensão controle pode ser compreendido como a capacidade decisória do profissional sobre o seu trabalho (MacKay et al., 2004). O apoio social dos colegas e do supervisor são avaliados separadamente na ICAO para aumentar a clareza das relações destes construtos com outras variáveis (MacKay et al., 2004). A dimensão papel investiga os índices de clareza de papel que os profissionais vivenciam. Desta forma, nessa dimensão avalia-se se os profissionais compreendem qual sua função na organização, quais atividades são englobas em seu cargo de trabalho e se os profissionais necessitam atender demandas que são conflitantes entre si (MacKay et al., 2004). A dimensão mudança avalia a comunicação dentro da empresa em situações de mudança, estimando a comunicação entre gestores e colaboradores (MacKay et al., 2004).

$\mathrm{Na}$ ICAO, a categoria demandas do trabalho é investigada com foco na carga de trabalho e no relacionamento entre colegas através das dimensões demanda e assédio moral. Assim, a dimensão demanda avalia a carga de trabalho e forma que as tarefas laborais são distribuídas e exigidas do profissional (MacKay et al., 2004). A dimensão assédio moral, orginalmente avaliada como relações no trabalho, investiga como os profissionais interagem no ambiente de trabalho, quais as práticas de trabalho promovidas e a presença de assédio moral no trabalho (MacKay et al., 2004).
Os achados apresentados demonstram a relação dos fatores psicossociais com desfechos positivos no trabalho (Toderi \& Balducci, 2015). Tendo em vista as evidências sobre as relações da demanda e dos recursos do trabalho com os recursos pessoais e o bem-estar no trabalho (Bakker \& Demerouti, 2017; Brunetto et al., 2013; Lysova, Allan, Dik, Duffy, \& Steger, 2019), investigamos as evidências de validade da ICAO por meio de sua relação com variáveis positivas de recursos pessoais (i.e. autoeficácia ocupacional), índices de bem-estar no trabalho (i.e., engajamento no trabalho) e recursos do trabalho (i.e., sentido no trabalho).

Os recursos pessoais têm mostrado diferentes associações com a demanda e os recursos do trabalho (Chan et al., 2017; Xanthopoulou et al., 2007). Nesse sentido, é provável que a autoeficácia ocupacional, como um recurso pessoal, tenha uma relação positiva com os recursos do trabalho (H1). Por fim, tem-se a hipótese que a autoeficácia ocupacional possui uma relação negativa com a demanda e o assédio moral (H2).

Estudos anteriores mostraram associações positivas entre os recursos do trabalho e o engajamento, ao passo que entre a demanda e o engajamento as associações encontradas foram negativas (Bakker, Hakanen, Demerouti, \& Xanthopoulou, 2007; Brunetto et al., 2013; Chen, 2016). Nessa perspectiva, espera-se uma associação positiva entre os recursos do trabalho com o engajamento (H3). Em contrapartida, é provável encontrar uma associação negativa para a demanda e o assédio moral com o engajamento (H4).

Fatores individuais, organizacionais e sociais interagem para promover um trabalho significativo (Lysova et al., 2019). Contemporaneamente, percebe-se um crescente interesse em carreiras que tenham significado e propósito para o indivíduo e que não represente, apenas, uma maneira de obter seu sustento. Dessa forma, o sentido do trabalho é um importante recurso do trabalho (Ahmed, Majid, \& Zin, 2016) que representa uma atividade laboral pessoalmente significativa (Steger, Dik, \& Duffy, 2012).

Estudos anteriores encontraram associações positivas entre os recursos do trabalho e o sentido do trabalho, em contrapartida, associações negativas entre a demanda e o sentido do trabalho (Berg, Dutton, \& Wrzesniewski, 2013; Lavoro, Gatti, \& Ghislieri, 2014). Dessa forma, é esperado que os recursos do trabalho se associem de maneira positiva com o sentido do trabalho (H5), como também a demanda e o assédio moral se associem de forma negativa com o sentido do trabalho (H6).

\section{Método}

\section{Participantes}

Foram utilizadas duas amostras distintas para avaliar a ICAO-35 e a ICAO-25. A amostra usada para o estudo da ICAO-35 contou com participantes de todas as regiões brasileiras (região Sul com 59,21\%; Sudeste com 28,09\%; Nordeste com 27,37\%; Centro-oeste com 9,1\% e Norte com 1,66\%). Para o estudo da ICAO-25, 94,1\% dos participantes declararam residir na região Sudeste e o restante nas demais regiões do país.

Para a ICAO-35, a amostra foi composta por 1.325 participantes, onde $63,8 \%(n=846)$ de mulheres e $36,2 \%(n=479)$ de homens, com idades entre 18 a 77 anos $(M=39,9 ; D P=11,9)$. Do total de participantes, $67,70 \%(n=897)$ se declararam casados ou em união estável. Referente à escolaridade, grande parte se declarou mestre $(40,60 \% ; n=538)$ ou doutor(a)/pós-doutor(a) $(30,34 \% ; n=402)$. Em relação a ocupação, os professores universitários representaram $47,90 \%(n=479)$, seguidos por profissionais de nível superior $(42,7 \% ; n=566)$, função de nível técnico $(8,7 \% ; n=115)$ e função de nível auxiliar $(0,7 \% ; n=9)$.

A segunda amostra, para a ICAO-25, foi composta por 
559 participantes com $55,1 \%$ de mulheres $(n=308)$ e $44,9 \%$ homens $(n=251)$, com idades entre 20 a 78 anos $(M=43,5$; $D P=12)$. Em relação ao estado civil, $58,32 \%(n=326)$ se declararam casados(as) ou em união estável Referente à escolaridade, $46,87 \%(n=262)$ possuía especialização. Em relação ao trabalho, $22 \%(n=123)$ se declararam professores universitários e 78\% $(n=436)$ exerciam ocupações diversificadas.

\section{Instrumentos}

Procedimentos de tradução e adaptação da ICAO. Inicialmente a ICAO foi traduzida por dois juízes brasileiros, fluentes em inglês, com especialização em psicometria ou em Psicologia Organizacional e do Trabalho. No processo de tradução, os juízes foram orientados a valorizar semelhança conceitual dos itens no processo, com o objetivo de possibilitar a equivalência entre o original e a versão brasileira (Borsa, Damásio, \& Bandeira, 2012). As duas versões traduzidas foram integradas na primeira versão em português. Após isso, a primeira versão da escala foi avaliada por um grupo focal $(n=12)$, o qual sugeriu adaptações a escrita dos itens. Após isso, foi realizada a tradução reversa da versão adaptada por um pesquisador bilíngue com conhecimento do construto. Ao comparar a versão brasileira traduzida e a original em inglês foi considerado que essas poderiam ser consideradas como equivalentes.

Instrumentos de coletas de dados. O Levantamento Sociodemográfico teve o intuito de identificar a amostra. Para tal, foram coletadas as seguintes variáveis: sexo, idade, estado civil, escolaridade, ocupação e região de residência.

A escala ICAO possui uma versão de 35 itens e 25 itens, distribuídos em sete dimensões: controle, papel, apoio social do supervisor, apoio social dos colegas, mudança, demanda e assédio moral (Cousins et al., 2004). A ICAO tem como objetivo investigar os níveis de recursos (p. ex., controle, clareza de papel e apoio social), demanda (p. ex., emocional, cognitiva ou física), assédio moral e mudança vivenciados pelos profissionais no ambiente de trabalho (MacKay et al., 2004). O instrumento é respondido em uma escala tipo Likert de cinco pontos, em que os extremos são "(1) Nunca” e "(5) Sempre”: "Eu posso escolher o que eu faço no meu trabalho" (controle); "Eu tenho clareza de quais são as minhas obrigações e responsabilidades" (papel); "Eu recebo retorno positivo (feedback) sobre o trabalho que eu faço" (apoio social do supervisor); "Se o trabalho for difícil, meus colegas me ajudarão" (apoio social dos colegas); "Quando são feitas mudanças no trabalho, eu sei bem como elas vão funcionar na prática” (mudança); "Eu sou pressionado a trabalhar por longas horas" (demanda) e "Eu sou alvo de assédio moral no trabalho" (assédio moral).

A autoeficácia ocupacional foi avaliada pela Escala de Autoeficácia Ocupacional - Versão Breve (EAO-VB). Desenvolvida por Rigotti, Schyns e Mohr (2008) e adaptada para o Brasil por Damásio, Freitas e Koller (2014) com alfa de Cronbach de 0,78. Composta por uma única dimensão distribuída em seis itens, respondidos em uma escala tipo Likert de cinco pontos, em que os extremos são “(1) Discordo totalmente” e “(5) Concordo": "Eu alcanço as metas que eu estabeleço para mim mesmo em meu trabalho". A consistência interna da escala no presente estudo foi adequada, com alfa de Cronbach de 0,82.

O engajamento no trabalho foi medido pela Escala Utrecht de Engajamento no Trabalho - UWES-9 (Schaufeli, Bakker, \& Salanova, 2006). A adaptação brasileira, com alfa de Cronbach de 0,93 e Confiabilidade Composta (CC) de 0,91, foi composta por nove itens, respondidos em uma escala tipo Likert de seis pontos que variam de (0) Nunca a (6) Sempre, distribuídos em três dimensões: vigor (três itens), dedicação (três itens) e absorção (três itens). Exemplo de item: "Em meu trabalho, sinto-me repleto (cheio) de energia" (Ferreira et al., 2016). O valor de consistência interna da escala foi satisfatório na presente amostra $(\alpha=0,93)$.

O sentido do trabalho foi avaliado pela Escala de Sentido no Trabalho (Steger et al., 2012). A versão brasileira (Leonardo, Pereira, Damásio, \& Valentini, 2019) é composta por dez itens em uma única dimensão, respondidos em uma escala tipo Likert de seis pontos: (1) totalmente falsa a (5) totalmente verdadeira. Exemplo de item: "Encontrei um trabalho realizador". A escala apresentou índices de Confiabilidade Composta de 0,94. Na presente amostra o valor de consistência interna adequado, com alfa de Cronbach de 0,95.

\section{Procedimentos de Coleta de Dados e Cuidados Éticos}

O presente estudo foi submetido e aprovado pelo Comitê de Ética em Pesquisa [CEP] - Plataforma Brasil com CAAE 65103317.6.0000.5289. Após a aprovação, a coleta de dados foi iniciada. Segundo a diretriz 466/12 e anteriores do Conselho Nacional de Saúde, para colaborar os participantes declararam sua concordância através do Termo de Consentimento Livre e Esclarecido, tendo garantido o sigilo e a confidencialidade sobre sua identidade. O formulário virtual foi desenvolvido através da plataforma Survey Monkey com os instrumentos citados anteriormente. Os participantes foram convidados por meio de lista de e-mails, grupos de mensagem instantânea de Whatsapp e da rede social Linkedin. Através de cada um desses mecanismos, foi enviado o link para acesso ao formulário.

\section{Procedimentos de Análise de Dados}

De forma a verificar qual melhor ajuste entre ICAO-35 e ICAO-25, analisou-se os índices: qui-quadrado $\left(\chi^{2}\right.$ - avalia a probabilidade do modelo teórico ser ajustado aos dados, quanto mais elevado for o $\chi^{2}$, pior será o ajuste ); Root Mean Square Error of Approximation (RMSEA - verifica a adequação ao modelo com valor esperado inferior a 0,08 , podendo ser aceito até 0,10 e considerando-se o intervalo de confiança de 90\%); Tucke$r$-Lewis Index e Comparative Fit Index (TLI e CFI - devem situar-se acima de 0,90, sendo desejável acima de 0,95) (Brown, 2015).

De forma a verificar a estrutura fatorial do instrumento, empregou-se análises fatoriais confirmatórias (AFC). Com o intuito de testar o ajuste da solução de sete fatores de primeira ordem para a ICAO-35 e ICAO-25 (controle, demanda, apoio social dos colegas, apoio social do supervisor, mudança, papel e assédio moral) do modelo, utilizou-se a AFC no programa MPlus (Muthén \& Muthén, 2015), cujo método de estimação foi WLSMV (Weighted Least Squares Mean - and Variance-adjusted). A escolha pelo método WLSMV se deu em razão dele usar de correlações policóricas (Holgado-Tello, Chacón-Moscoso, Barbero-García, \& Vila-Abad, 2010).

As evidências de validade baseadas nas medidas externas (validade convergente) foram desenvolvidas através da modelagem de equações estruturais (MEE), pelo método de estimação WLSMV. Buscou-se avaliar a relação das dimensões das escalas ICAO-35 e ICAO-25 com as variáveis engajamento, sentido do trabalho e autoeficácia ocupacional. A consistência interna da ICAO-35 e ICAO-25 foi avaliada utilizando o coeficiente de fidedignidade composta (fc) e o alfa de Cronbach.

\section{Resultados}

As evidências de estabilidade para solução de sete fatores de primeira ordem para a ICAO-35 e ICAO-25 (Edwards \& Webster, 2012) motivaram a avaliação desta solução para a versão brasileira da escala. Conforme proposto teoricamente (Cousins et al., 
2004), os itens da ICAO-35 e ICAO-25 apresentaram cargas fatoriais adequadas em suas respectivas dimensões (ver Tabela 1). A análise das cargas fatoriais demonstra que os itens apresentam precisão, pois se mostraram robustos, com cargas fatoriais variando de 0,51 a 0,95 para a ICAO-35 e de 0,51 a 0,97 para a ICAO-25.

Foi observado que o modelo de sete fatores de primeira ordem obteve índices de ajuste $\left(\chi^{2}(g)\right.$, CFI, TLI e RMSEA) satisfatórios em ambas versões (ver Tabela 1). Porém, observou-se que a ICAO-25 obteve índices superiores a ICAO-35, com exceção dos valores de resíduos (RMSEA). Os índices de confiabilidade foram avaliados por meio do coeficiente de fidedignidade composta $(f c)$ e alfa de Cronbach. Tais índices foram adequados tanto para as dimensões da ICAO de 35 itens, como para sua forma reduzida (ver Tabela 1).

As versões da ICAO apresentaram dimensões relacionadas entre si de forma significativa e na direção esperada. Foi observado que os recursos estiveram positivamente relacionados entre si (controle, papel, mudança, apoio social dos colegas e supervisor) e negativamente com a demanda e assédio moral. Ressalta-

Tabela 1

Análise fatorial confirmatória da estrutura de sete fatores de primeira ordem da ICAO-35 e ICAO-25 e indices de ajustes

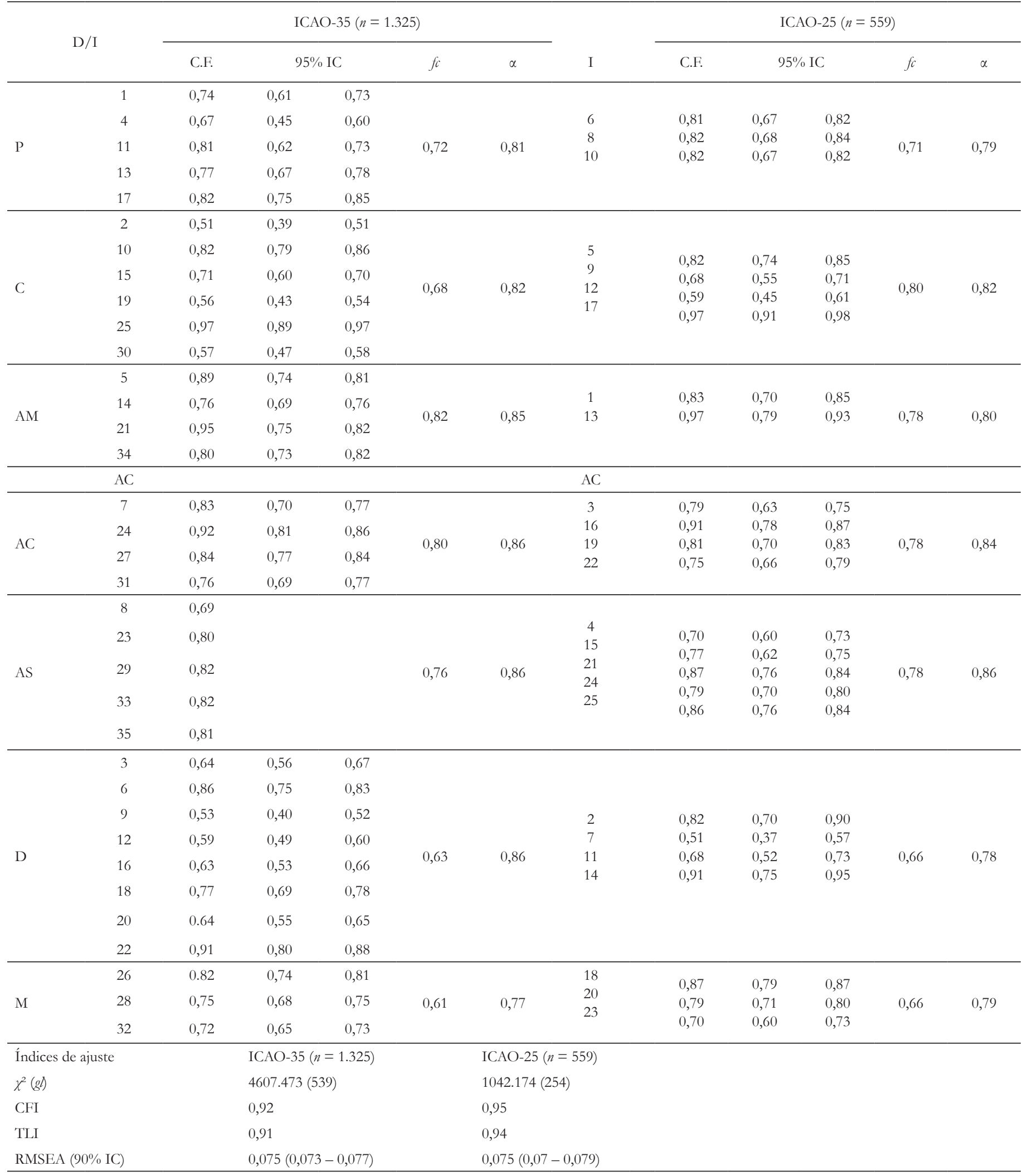

Nota. Todos os itens foram significativos em $p<0,0001$. D/I = Dimensão/Item; C.F. = carga fatorial; $95 \%$ IC CF $=95 \%$ do Intervalo de Confiança da Carga Fatorial; $f c=$ confiabilidade composta; $\alpha=$ alfa de Cronbach; $n=$ número de participantes; $\mathrm{D}=$ Demanda; $\mathrm{C}=$ Controle; ASS = Apoio Social do Supervisor; ASC = Apoio Social dos Colegas; $\mathrm{P}=$ Papel; $\mathrm{M}=\mathrm{Mudança}$ $\mathrm{AM}=$ Assédio Moral. AEO = Autoeficácia Ocupacional, EnT = Engajamento no Trabalho; $\mathrm{ST}=$ Sentido no Trabalho. 
-se que, para a ICAO-25, as associações não foram significativas entre papel e apoio dos colegas, papel e assédio moral, apoio dos colegas e demanda. As dimensões demanda e assédio moral estiveram positivamente associados (ver Tabela 2 e Tabela 3). colegas e apoio social do supervisor com autoeficácia ocupacional se mostraram positivas e de baixa magnitude para a ICAO35. Ressalta-se que, para a ICAO-25, essas mesmas relações não foram significativas (ver Tabela 2). Em relação ao assédio moral

Tabela 2

Correlações entre as dimensões da ICAO-35 e entre as dimensões e variáveis externas

\begin{tabular}{|c|c|c|c|c|c|c|c|c|c|}
\hline Dimensões & 1 & 2 & 3 & 4 & 5 & 6 & 7 & 8 & 9 \\
\hline \multicolumn{10}{|l|}{ 1. Papel } \\
\hline \multirow{2}{*}{ 2. Controle } & $0,54 * *$ & & & & & & & & \\
\hline & $(0,50 ; 0,58)$ & & & & & & & & \\
\hline \multirow{2}{*}{ 3.Assédio Moral } & $-0,37 * *$ & $-0,51 * *$ & & & & & & & \\
\hline & $(-0,42 ;-0,32)$ & $(-0,55 ;-0,47)$ & & & & & & & \\
\hline \multirow{2}{*}{ 4. Apoio dos Colegas } & $0,42 * *$ & $0,58^{* *}$ & $-0,74 * *$ & & & & & & \\
\hline & $(0,37 ; 0,46)$ & $(0,54 ; 0,61)$ & $(-0,77 ;-0,72)$ & & & & & & \\
\hline \multirow{2}{*}{ 5. Apoio do Supervisor } & $0,54 * *$ & $0,71 * *$ & $-0,73 * *$ & $0,85^{* *}$ & & & & & \\
\hline & $(0,49 ; 0,58)$ & $(0,68 ; 0,74)$ & $(-0,75 ;-0,70)$ & $(0,84 ; 0,87)$ & & & & & \\
\hline \multirow{2}{*}{ 6. Demanda } & $-0,32 * *$ & $-0,41^{* *}$ & $0,68^{* *}$ & $-0,49 * *$ & $-0,50 * *$ & & & & \\
\hline & $(-0,37 ;-0,27)$ & $(-0,46 ;-0,36)$ & $(0,64 ; 0,71)$ & $(-0,53 ;-0,44)$ & $(-0,54 ;-0,46)$ & & & & \\
\hline \multirow{2}{*}{ 7. Mudança } & $0,62 * *$ & $0,84 * *$ & $-0,66 * *$ & $0,75^{* *}$ & $0,94 * *$ & $-0,43 * *$ & & & \\
\hline & $(0,59 ; 0,66)$ & $(0,82 ; 0,85)$ & $(-0,69 ;-0,63)$ & $(0,72 ; 0,77)$ & $(0,93 ; 0,94)$ & $(-0,48 ;-0,38)$ & & & \\
\hline \multirow{2}{*}{$\begin{array}{l}\text { 8. Autoeficácia } \\
\text { Ocupacional }\end{array}$} & $0,68^{* *}$ & $0,51 * *$ & $-0,37 * *$ & 0,36 ** & $0,44^{* *}$ & $-0,29 * *$ & $0,48^{* *}$ & & \\
\hline & $(0,64 ; 0,71)$ & $(0,47 ; 0,55)$ & $(-0,42 ;-0,32)$ & $(0,31 ; 0,41)$ & $(0,39 ; 0,49)$ & $(-0,34 ;-0,23)$ & $(0,43 ; 0,52)$ & & \\
\hline \multirow{2}{*}{$\begin{array}{l}\text { 9. Engajamento no } \\
\text { Trabalho }\end{array}$} & $0,44^{* *}$ & $0,55^{* *}$ & $-0,36^{* *}$ & $0,32^{* *}$ & $0,45^{* *}$ & $-0,16^{* *}$ & $0,51 * *$ & $0,58^{* *}$ & \\
\hline & $(0,39 ; 0,48)$ & $(0,51 ; 0,59)$ & $(-0,41 ;-0,31)$ & $(0,27 ; 0,37)$ & $(0,41 ; 0,50)$ & $(-0,22 ;-0,11)$ & $(0,46 ; 0,55)$ & $(0,54 ; 0,62)$ & \\
\hline \multirow{2}{*}{ 10.Sentido do Trabalho } & $0,48^{* *}$ & $0,49 * *$ & $-0,29 * *$ & $0,29 * *$ & $0,44 * *$ & $-0,16^{* *}$ & $0,47 * *$ & $0,56^{* *}$ & $0,84^{* *}$ \\
\hline & $(0,44 ; 0,53)$ & $(0,45 ; 0,54)$ & $(-0,35 ;-0,24)$ & $(0,23 ; 0,34)$ & $(0,39 ; 0,48)$ & $(-0,21 ;-0,10)$ & $(0,42 ; 0,51)$ & $(0,52 ; 0,60)$ & $(0,82 ; 0,85)$ \\
\hline
\end{tabular}

Nota. $* p<0,010$; Valores em parênteses representam o intervalo de $95 \%$ da correlação.

As associações entre os recursos papel e controle com a variável externa autoeficácia ocupacional foram positivas e de magnitude moderada. Entretanto, a associação do recurso mudança com a autoeficácia ocupacional se apresentou positiva e de magnitude moderada apenas para a ICAO-35 e com baixa magnitude para a ICAO-25 (ver Tabela 2 e Tabela 3).

Além disso, as associações dos recursos apoio social dos e demanda, as associações com a autoeficácia ocupacional foram negativas e de baixa magnitude. Porém, para a ICAO-25, essas relações não foram significativas (ver Tabela 2 e Tabela 3).

Os recursos do trabalho apresentaram associações positivas com o engajamento, porém as magnitudes variaram entre moderada e baixa. Para a ICAO-35 as associações encontradas entre os recursos papel, apoio social dos colegas e apoio social do supervisor

Tabela 3

Correlações entre as dimensões da ICAO-25 e entre as dimensões e variáveis externas

\begin{tabular}{|c|c|c|c|c|c|c|c|c|c|}
\hline Dimensões & 1 & 2 & 3 & 4 & 5 & 6 & 7 & 8 & 9 \\
\hline \multicolumn{10}{|l|}{ 1. Papel } \\
\hline \multirow{2}{*}{ 2. Controle } & $0,54 * *$ & & & & & & & & \\
\hline & $(0,40 ; 0,66)$ & & & & & & & & \\
\hline \multirow{2}{*}{ 3.Assédio Moral } & $-0,23 *$ & $-0,45^{* *}$ & & & & & & & \\
\hline & $(-0,39 ;-0,05)$ & $(-0,58 ;-0,30)$ & & & & & & & \\
\hline \multirow{2}{*}{ 4. Apoio dos Colegas } & $0,26 * *$ & $0,53^{* *}$ & $-0,58^{* *}$ & & & & & & \\
\hline & $(0,08 ; 0,42)$ & $(0,38 ; 0,64)$ & $(-0,69 ;-0,44)$ & & & & & & \\
\hline \multirow{2}{*}{ 5. Apoio do Supervisor } & $0,62 * *$ & $0,67 * *$ & $-0,50 * *$ & $0,73^{* *}$ & & & & & \\
\hline & $(0,50 ; 0,72)$ & $(0,56 ; 0,76)$ & $(-0,62 ;-0,35)$ & $(0,64 ; 0,81)$ & & & & & \\
\hline \multirow{2}{*}{ 6. Demanda } & $-0,43^{* *}$ & $-0,46^{* *}$ & $0,70^{* *}$ & $-0,21 *$ & $-0,57 * *$ & & & & \\
\hline & $(-0,57 ;-0,27)$ & $(-0,59 ;-0,31)$ & $(0,60 ; 0,78)$ & $(-0,38 ;-0,04)$ & $(-0,68 ;-0,43)$ & & & & \\
\hline \multirow{2}{*}{ 7. Mudança } & $0,77 * *$ & $0,81 * *$ & $-0,41^{* *}$ & $0,56^{* *}$ & $0,89 * *$ & $-0,47 * *$ & & & \\
\hline & $(0,68 ; 0,83)$ & $(0,74 ; 0,87)$ & $(-0,55 ;-0,24)$ & $(0,42 ; 0,67)$ & $(0,84 ; 0,92)$ & $(-0,60 ;-0,32)$ & & & \\
\hline \multirow{2}{*}{$\begin{array}{l}\text { 8. Autoeficácia } \\
\text { Ocupacional }\end{array}$} & $0,72^{* *}$ & $0,51 * *$ & $-0,08$ & 0,05 & $0,29 * *$ & $-0,26 * *$ & $0,46^{* *}$ & & \\
\hline & $(0,62 ; 0,80)$ & $(0,37 ; 0,64)$ & $(-0,25 ; 0,10)$ & $(-0,13 ; 0,23)$ & $(0,12 ; 0,45)$ & $(-0,42 ;-0,09)$ & $(0,31 ; 0,59)$ & & \\
\hline \multirow{2}{*}{$\begin{array}{l}\text { 9. Engajamento no } \\
\text { Trabalho }\end{array}$} & $0,51 * *$ & $0,56^{* *}$ & $-0,28^{* *}$ & $0,38^{* *}$ & $0,39 * *$ & $-0,40 * *$ & $0,46^{* *}$ & $0,64 * *$ & \\
\hline & $(0,36 ; 0,63)$ & $(0,42 ; 0,67)$ & $(-0,44 ;-0,11)$ & $(0,22 ; 0,53)$ & $(0,23 ; 0,53)$ & $(-0,54 ;-0,24)$ & $(0,30 ; 0,59)$ & $(0,52 ; 0,74)$ & \\
\hline \multirow{2}{*}{ 10.Sentido do Trabalho } & $0,34 * *$ & $0,26 * *$ & $-0,04$ & 0,08 & 0,13 & $-0,23 * *$ & $0,24 * *$ & $0,57 * *$ & $0,72^{* *}$ \\
\hline & $(0,17 ; 0,49)$ & $(0,08 ; 0,42)$ & $(-0,22 ; 0,14)$ & $(-0,10 ; 0,25)$ & $(-0,05 ; 0,30)$ & $(-0,40 ;-0,06)$ & $(0,06 ; 0,40)$ & $(0,44 ; 0,68)$ & $(0,62 ; 0,80)$ \\
\hline
\end{tabular}

Nota. $* p<0,010$; Valores em parênteses representam o intervalo de $95 \%$ da correlação. 
com o engajamento foram positivas e de baixa magnitude, bem como positivas e de magnitude moderada entre os recursos controle e mudança com o engajamento. Para a ICAO-25, as associações encontradas entre os recursos papel, controle, apoio social do supervisor e mudança com o engajamento apresentaram associações positivas e de magnitude moderada. Contudo, somente a associação entre o recurso apoio social dos colegas e engajamento apresentou associação positiva e de baixa magnitude (ver Tabela 2 e Tabela 3).

As associações encontradas entre demanda e assédio moral com engajamento foram negativas e de baixa magnitude, na ICAO-35. Para a ICAO-25, a relação da demanda com o engajamento foi negativa e de magnitude moderada, enquanto a relação entre assédio moral com o engajamento foi negativa e de baixa magnitude (ver Tabela 2 e Tabela 3).

Os recursos controle, papel, apoio social do supervisor e mudança apresentaram associações positivas de magnitude moderada com o sentido do trabalho, na ICAO-35. Mas, na versão de 25 itens, os recursos controle e papel apresentaram associações positivas de baixa magnitude com o sentido do trabalho, enquanto as demais dimensões de recursos não apresentaram relações estatisticamente significativas (ver Tabela 2 e Tabela 3).

Em relação às associações entre demanda e assédio moral com o sentido do trabalho, os resultados mostraram associações negativas e de baixa magnitude para as versões de 35 e 25 itens. Porém, para a versão reduzida 25 itens, a associação entre assédio moral e sentido do trabalho não alcançou significância estatística (ver Tabela 2 e Tabela 3).

\section{Discussão}

O presente trabalho teve como objetivo adaptar e reunir evidências iniciais de validade da dimensionalidade, relações com as variáveis externas e fidedignidade no contexto brasileiro para a ICAO-35 e ICAO-25. A análise fatorial confirmatória apresentou índices de ajuste adequados na solução multifatorial de sete fatores de primeira ordem em ambas versões. Esses resultados confirmam os achados de Edwards e Webster (2012) para a versão inglesa das escalas, como também os estudos de Toderi et al. (2013) e Cassar, Bezzina e Buttigieg (2018) com profissionais italianos e malteses, respectivamente.

Os resultados demonstram que a ICAO-25 e ICAO35 constituem-se instrumentos adequados para avaliar as características do contexto organizacional. É importante ressaltar que em relação à brevidade da ICAO-25 e aos índices de ajustes, a ICAO-25 se mostrou superior a ICAO-35, exceto para os valores de resíduos. Desta forma, recomenda-se o uso da ICAO-25, quando comparada à ICAO-35.

Referente as evidências de fidedignidade, os índices de consistência interna para as dimensões da ICAO-35 e ICAO25 apresentaram alta magnitude, variando de 0,77 e 0,86 . Esses achados são semelhantes aos encontrados por Edwards e Webster (2012), os quais apresentaram valore entre 0,81 e 0,89. Além do alfa de Cronbach, a confiabilidade das dimensões foi medida por meio da fidedignidade composta $(f c)$. Os resultados achados foram adequados para as dimensões da escala com 35 e 25 itens.

As dimensões da escala ICAO-35 relacionaram-se entre si de forma significativa, como também na direção esperada. Resultado semelhante foi encontrado para a escala ICAO-25, com exceção para a relação entre demanda e apoio social dos colegas, a qual não se mostrou estatisticamente significativa. Ademais, para a ICAO35 e ICAO-25, encontrou-se que os recursos do trabalho se relacionaram positivamente entre si (controle, papel, mudança, apoio social dos colegas e apoio social supervisor), assim como demanda e assédio moral também estiveram positivamente associadas. Es- ses resultados vão de encontro a estudos anteriores, onde recursos referentes à organização, ao grupo de trabalho e organização da tarefa se relacionaram de maneira positiva, como também diferentes demandas do trabalho se associam positivamente entre si (Airila et al., 2014; Kulikowski \& Orzechowski, 2018). As relações positivas entre os recursos corroboram que ambientes com maior disponibilidade de recursos podem promover o processo motivacional (Bakker \& Demerouti, 2017; Schaufeli \& Taris, 2014). Desta forma, a presença de um recurso colabora para o desenvolvimento e fortalecimento de outros recursos do trabalho, criando um ciclo de aumento de recursos para os profissionais.

Por fim, os recursos do trabalho relacionaram-se negativamente com as dimensões demanda e assédio moral. Para a teoria JD-R, recursos e demanda constituem categorias diferentes e, consequentemente, levam a processos distintos. Dessa forma, enquanto o processo motivacional é caracterizado por elevados recursos que podem se refletir em mais motivação e melhor desempenho no trabalho, o processo de adoecimento conta com altas demanda de obstáculo que podem levar implicações para a saúde e à tensão ocupacional. Tais achados corroboram estudos que examinaram as relações entre recursos do trabalho e demandas de obstáculo (Bakker \& Demerouti, 2017; Schaufeli \& Taris, 2014)

A autoeficácia ocupacional associou-se positivamente aos recursos do trabalho e negativamente com demanda e assédio moral, confirmando as hipóteses H1 e H2. Desta forma, tais resultados convergem os achados de estudos anteriores sobre o tema, nos quais profissionais com maiores níveis de autoeficácia podem perceber uma maior abundância de recursos no trabalho, assim como a autoeficácia pode minimizar os efeitos negativos das demandas no trabalho (Brunner, Igic, Keller, \& Wieser, 2019; Lo Presti \& Nonnis, 2014).

Os recursos do trabalho associaram-se de forma positiva com o engajamento, por sua vez a demanda e o assédio moral associaram-se negativamente com engajamento, confirmando as hipóteses H3 e H4. Nesse sentido, os achados convergem para estudos encontrados na literatura, que demonstram que ambientes com abundância de recursos e baixos níveis de demanda e assédio propiciam o engajamento no trabalho (Bakker et al., 2007; Chen, 2016).

Por fim, as hipóteses H5 e H6 confirmaram o direcionamento das associações, sendo que os recursos do trabalho se associaram de forma positiva com o sentido do trabalho, enquanto as dimensões demanda e assédio moral associaram-se negativamente com o sentido do trabalho. Os achados corroboram estudos anteriores que demonstraram como as percepções de sentido do trabalho podem enriquecer a relação dos trabalhadores com os recursos do trabalho e, consequentemente, favorecer o envolvimento com o trabalho, como também diminuir os impactos das demandas de trabalho (Ahmed, Abdul Majid, Al-Aali, \& Mozammel, 2018; Fletcher, Bailey, \& Gilman, 2018).

O impacto das condições de trabalho ao bem-estar, desempenho e condições de saúde dos profissionais expõe a relevância de serem disponibilizados instrumentos que avaliem essas características de forma precisa (Bakker \& Demerouti, 2017). O presente estudo teve como principal contribuição a validação das escalas ICAO-35 e ICAO-25, para o contexto brasileiro. Recomenda-se o uso da ICAO-25 em detrimento da ICAO-35, devido aos índices de ajustes achados, como também pela brevidade da escala. O uso de escalas breves na avaliação das condições de trabalho, pode auxiliar na construção de protocolos menos extensos principalmente em estudos que avaliam simultaneamente diversas características laborais. O uso de escalas breves pode ser vantajoso para evitar a exaustão dos participantes ao responderem protocolos de pesquisa demasiadamente extensos (Damásio et al., 2014).

Sobre as limitações deste estudo, é possível que os da- 
dos tenham sofrido o efeito de desejabilidade social, por terem sido utilizados exclusivamente instrumentos de autorrelato. Outra questão importante a ser mencionado é o fato de as amostras serem por conveniência, sendo que a maior parte dos participantes apresentava alta escolaridade.

As limitações do presente estudo expõem que é imprescindível a avaliação das propriedades psicométricas da ICAO em amostras de trabalhadores pertencentes a diversos setores e organizações. Em razão disso, sugere-se que estudos futuros investiguem a elaboração de itens específicos para os diferentes grupos laborais como, por exemplo, professores e profissionais da saúde. A compreensão em profundidade das demandas e recursos do trabalho desses grupos laborais permitirá que sejam propostas ações para prevenir o adoecimento profissional, bem como intervenções para a promoção do bem-estar dos profissionais. Do ponto de vista da prática, as organizações necessitam identificar as demandas de obstáculo e as demandas desafiadoras, bem como a variedade de recursos estruturais e sociais disponíveis. A avaliação das condições de trabalho é uma ação tática que precede qualquer iniciativa para a construção de ambientes de trabalho saudáveis.

\section{Referências}

Ahmed, U., Abdul Majid, A. H., Al-Aali, L., \& Mozammel, S. (2018). Can meaningful work really moderate the relationship between supervisor support, coworker support and work Engagement? Management Science Letters, 9(2018), 229-242. https://doi.org/10.5267/i.msl.2018.11.016

Ahmed, U., Majid, A. H. A., \& Zin, M. L. M. (2016). Meaningful Work and Work Engagement: A Relationship Demanding Urgent Attention. International Journal of Academic Research in Business and Social Sciences, 6(8), 116-122. https://doi.org/10.6007/IJARBSS/v6-i8/2264

Airila, A., Hakanen, J. J., Schaufeli, W. B., Luukkonen, R., Punakallio, A., \& Lusa, S. (2014). Are job and personal resources associated with work ability 10 years later? The mediating role of work engagement. Work \& Stress, 28(1), 87-105. https://doi.org/10.1080/02678373.2013.872208

Bakker, A. B., \& Demerouti, E. (2007). The Job Demands-Resources model: State of the art. Journal of Managerial Psychology, 22(3), 309-328. https://doi. org/10.1108/02683940710733115

Bakker, A. B., \& Demerouti, E. (2014). Job Demands-Resources Theory. Em P. Y. Chen \& C. L. Cooper (Eds.), Work and Wellbeing: Wellbeing: A Complete Reference Guide (pp. 1-28). https://doi.org/10.1002/9781118539415. wbwell019

Bakker, A. B., \& Demerouti, E. (2017). Job demands-resources theory: taking stock and looking forward. Journal of Occupational Health Psychology, 22(3), 273-285. https://doi.org/ 10.1037/ocp0000056

Bakker, A. B., \& Demerouti, E. (2018). Multiple levels in job demands-resources theory: Implications for employee well-being and performance. Em E. Diener, S. Oishi, \& L. Tay (Eds.), Handbook of well-being (pp. 1-13). Salt Lake City, UT: DEF Publishers

Bakker, A. B., Demerouti, E., de Boer, E., \& Schaufeli, W. B. (2003). Job demands and job resources as predictors of absence duration and frequency. Journal of Vocational Behavior, 62(2), 341-356. https://doi.org/10.1016/S00018791(02)00030-1

Bakker, A. B., Demerouti, E., \& Sanz-Vergel, A. I. (2014). Burnout and work engagement: The JD-R approach. Annual Review of Organizational Psychology and Organizational Behavior, 1(1), 389-411. https://doi.org/10.1146/annurevorgpsych-031413-091235

Bakker, A. B., Hakanen, J. J., Demerouti, E., \& Xanthopoulou, D. (2007). Job resources boost work engagement, particularly when job demands are high. Journal of Educational Psychology, 99(2), 274-284. https://doi. org/10.1037/0022-0663.99.2.274

Berg, J. M., Dutton, J. E., \& Wrzesniewski, A. (2013). Job crafting and meaningful work. Em B. J. Dik, Z. S. Byrne, \& M. F. Steger (Eds.), Purpose and meaning in the workplace (pp. 81-104). Washington, DC: American Psychological Association

Borsa, J. C., Damásio, B. F., \& Bandeira, D. R. (2012). Adaptação e validação de instrumentos psicológicos entre culturas: algumas considerações. Paidéia (Ribeirão Preto), 22(53), 423-432. https://doi.org/10.1590/198243272253201314

Brown, T. A. (2015). Confirmatory factor analysis for applied research ( $2^{\mathrm{a}} \mathrm{ed}$.). New York: The Guilford Press.
Brunetto, Y., Xerri, M., Shriberg, A., Farr-Wharton, R., Shacklock, K., Newman, S., \& Dienger, J. (2013). The impact of workplace relationships on engagement, well-being, commitment and turnover for nurses in Australia and the USA. Journal of Advanced Nursing, 69(12), 2786-2799. https://doi. org $/ 10.1111 /$ jan. 12165

Brunner, B., Igic, I., Keller, A. C., \& Wieser, S. (2019). Who gains the most from improving working conditions? Health-related absenteeism and presenteeism due to stress at work. The European Journal of Health Economics, 20(8), 1165-1180. https://doi.org/10.1007/s10198-019-01084-9

Cassar, V., Bezzina, F., \& Buttigieg, S. (2018). Investigating the psychometric properties and assessment capabilities of the short version of the Health and Safety Executive's Management Standards Indicator Tool. International Journal of Human Resource Management, 5192, 1-26. https://doi.org/10.1080/ $\underline{09585192.2018 .1431955}$

Chan, X. W., Kalliath, T., Brough, P., O’Driscoll, M., Siu, O.-L., \& Timms, C. (2017). Self-efficacy and work engagement: test of a chain model. International Journal of Manpower, 38(6), 819-834. https://doi.org/10.1108/ IJM-11-2015-0189

Chen, I.-S. (2016). Work engagement and its antecedents and consequences: A case of lecturers teaching synchronous distance education courses. Computers in Human Behavior, 72, 655-663. https://doi.org/10.1016/j.chb.2016.10.002

Costa, M. T. P., Borges, L. de O., \& Barros, S. C. (2015). Condições de trabalho e saúde psíquica: um estudo em dois hospitais universitários. Revista Psicologia Organizações e Trabalho, 15(1), 43-58. https://doi.org/10.17652/ rpot/2015.1.490

Cousins, R., MacKay, C. J., Clarke, S. D., Kelly, C., Kelly, P. J., \& McCaig, R. H (2004). 'Management Standards' work-related stress in the UK: practical development. Work \& Stress, 18(2), 113-136. https://doi.org/10.1080/0267 8370410001734322

Crawford, E. R., LePine, J. A., \& Rich, B. L. (2010). Linking job demands and resources to employee engagement and burnout: A theoretical extension and meta-analytic test. Journal of Applied Psychology, 95(5), 834-848. https:// doi.org/10.1037/a0019364

Damásio, B. F., Freitas, C. P. P. de, \& Koller, S. H. (2014). Occupational self-efficacy Scale - Short Form (OSS-SF): adaptation and evidence of construct validity of the Brazilian version. Revista Brasileira de Orientacao Profissional, 15(1), 65-74. Recuperado de http://pepsic.bvsalud.org/scielo. php? script $=$ sci arttext\&pid $=$ S1679-33902014000100008\&lng=pt\&nrm=iso

Demerouti, E., \& Bakker, A. B. (2011). The Job Demands-Resources model: Challenges for future research. SA Journal of Industrial Psychology, 37(2), 1-9. https://doi.org/10.4102/saiip.v37i2.974

Demerouti, E., Bakker, A. B., Nachreiner, F., \& Schaufeli, W. B. (2001). The job demands-resources model of burnout. Journal of Applied Psychology, 86(3), 499-512. https://doi.org/ 10.1037/0021-9010.86.3.499

Edwards, J. A., \& Webster, S. (2012). Psychosocial risk assessment: Measurement invariance of the UK Health and Safety Executive's Management Standards Indicator Tool across public and private sector organizations (Vol. 26). https://doi.org/10.1080/02678373.2012.688554

Ferreira, M. C., Valentini, F., Damásio, B. F., Mourão, L., Porto, J. B., Chinelato, R. S. de C., ... Pereira, M. M. (2016). Evidências adicionais de validade da UWES-9 em amostras brasileiras. Estudos de Psicologia, 21(4), 435-445. https://doi.org/10.5935/1678-4669.20160042

Fletcher, L., Bailey, C., \& Gilman, M. W. (2018). Fluctuating levels of personal role engagement within the working day: A multilevel study. Human Resource Management Journal, 28(1), 128-147. https://doi.org/10.1111/1748$\underline{8583.12168}$

Gouvêa, L. A. V. N. de. (2016). As condições de trabalho e o adoecimento de professores na agenda de uma entidade sindical. Saúde Debate, 40(111), 206-219. https://doi.org/10.1590/0103-1104201611116

Hakanen, J. J., Bakker, A. B., \& Schaufeli, W. B. (2006). Burnout and work engagement among teachers. Journal of School Psychology, 43(6), 495-513. https://doi.org/10.1016/i.jsp.2005.11.001

Hakanen, J. J., \& Roodt, G. (2010). Using the job demands-resources model to predict engagement: Analysing a conceptual model. Em A. B. Bakker \& M. P. Leiter (Eds.), Work Engagement: A Handbook of Essential Theory and Research (pp. 85-101). https://doi.org/10.4324/9780203853047

Holgado-Tello, F. P., Chacón-Moscoso, S., Barbero-García, I., \& Vila-Abad, E. (2010). Polychoric versus Pearson correlations in exploratory and confirmatory factor analysis of ordinal variables. Quality and Quantity, 44(1), 153-166. https://doi.org/10.1007/s11135-008-9190-y

Kulikowski, K., \& Orzechowski, J. (2018). All employees need job resources Testing the Job Demands-Resources Theory among employees with either high or low working memory and fluid intelligence. Medycyna Pracy, 69(5), 1-14. https://doi.org/10.13075/mp.5893.00709

Lavoro, C. G., Gatti, P., \& Ghislieri, C. (2014). Richieste lavorative, significatività percepita del lavoro e intenzione di turnover degli infermieri. Med Lav, 105(1), 37-47. Recuperado de https://iris.unito.it/retrieve/ handle/2318/148187/23514/MedLavCGC 2014.pdf 
Leonardo, M. da G., Pereira, M. M., Damásio, B. F., \& Valentini, F. (2019). Adaptação do Inventário de Sentido do Trabalho (WAMI) para o contexto brasileiro. Revista Brasileira de Orientação Profissional, 20(1), 79-89. https://doi. org/10.26707/1984-7270/2019v20n1p79

Lo Presti, A., \& Nonnis, M. (2014). Testing the job demands-resources model: Evidence from a sample of italian employees. TPM - Testing, Psychometrics, Methodology in Applied Psychology, 21(1), 89-101. https://doi.org/10.4473/ TPM21.1.6

Lysova, E. I., Allan, B. A., Dik, B. J., Duffy, R. D., \& Steger, M. F. (2019). Fostering meaningful work in organizations: A multi-level review and integration. Journal of Vocational Behavior, 110, 374-389. https://doi. org/10.1016/i.jvb.2018.07.004

MacKay, C. J., Cousins, R., Kelly, P. J., Lee, S., \& McCaig, R. H. (2004). 'Management Standards' and work-related stress in the UK: Policy background and science. Work \& Stress, 18(2), 91-112. https://doi.org/10.1 $\underline{080 / 02678370410001727474}$

Muthén, L. K., \& Muthén, B. O. (2015). Mplus user's guide: statistical analysis with latent variables ( $7^{\mathrm{a}} \mathrm{ed}$.). Recuperado de https://www.statmodel.com/download/usersguide/MplusUserGuideVer 7.pdf

Nahrgang, J. D., Morgeson, F. P., \& Hofmann, D. A. (2011). Safety at work: A meta-analytic investigation of the link between job demands, job resources, burnout, engagement, and safety outcomes. Journal of Applied Psychology, 96(1), 71-94. https://doi.org/10.1037/a0021484

Rigotti, T., Schyns, B., \& Mohr, G. (2008). A Short Version of the Occupational Self-Efficacy Scale: Structural and Construct Validity Across Five Countries. Journal of Career Assessment, 16(2), 238-255. https://doi. org/10.1177/1069072707305763

Schaufeli, W. B., Bakker, A. B., \& Salanova, M. (2006). The Measurement of Short Questionnaire: A Cross-National Study UWES-9. Educational and Psychological Measurement, 66(4), 701-716. https://doi.org/10.1177/0013164405282471

Schaufeli, W. B. (2017). Applying the Job Demands-Resources model: A 'how to' guide to measuring and tackling work engagement and burnout. Organizational Dynamics, 46(2), 120-132. https://doi.org/10.1016/i.orgdyn.2017.04.008

Schaufeli, W. B., \& Taris, T. W. (2014). A Critical review of the Job Demands-Resources Model: implications for improving work and health. Em G. F. Bauer \& O. Hämmig (Eds.), Bridging Occupational, Organizational and Public Health (Vol. 9789400756, pp. 43-68). https://doi.org/10.1007/978-94-007-5640$\underline{34}$

Steger, M. F., Dik, B. J., \& Duffy, R. D. (2012). Measuring Meaningful Work: The Work and Meaning Inventory (WAMI). Journal of Career Assessment, 20(3), 322-337. https://doi.org/10.1177/1069072711436160

Toderi, S., Balducci, C., Edwards, J. A., Sarchielli, G., Broccoli, M., \& Mancini, G. (2013). Psychometric properties of the UK and Italian Versions of the HSE stress indicator tool. European Journal of Psychological Assessment, 29(1), 72-79. https://doi.org/10.1027/1015-5759/a000122

Toderi, S. \& Balducci, C. (2015). HSE management standards indicator tool and positive work-related outcomes. International Journal of Workplace Health $\mathrm{Ma}$ nagement, 8(2), 92-108. https://doi.org/10.1108/IJWHM-11-2013-0044

Xanthopoulou, D., Bakker, A. B., Demerouti, E., \& Schaufeli, W. B. (2007). The role of personal resources in the job demands-resources model. International Journal of Stress Management, 14(2), 121-141. https://doi.org/10.1037/1072$\underline{5245.14 .2 .121}$

\section{Informações sobre os autores:}

\section{Paula Andréa Prata Ferreira}

Rua Mal. Deodoro, 217 - Bloco A - Centro, Niterói - RJ, Brasil, CEP 24030-060.E-mail: paulaprata@gmail.com

\section{Clarissa Pinto Pizarro de Freitas}

E-mail: freitas.cpp@gmail.com

\section{Rita Pimenta de Devotto}

E-mail: rpimentad@gmail.com

\section{Bruno Figueiredo Damásio}

E-mail: bf.damasio@gmail.com 